\title{
Berberine exerts an anti-inflammatory role in ocular Behcet's disease
}

\author{
YAN YANG, QIN WANG, MANYUN XIE, PING LIU, XIN QI, XIAO LIU and ZHUO LI \\ Department of Ophthalmology, The Second Xiangya Hospital, Central South University, \\ Changsha, Hunan 410010, P.R. China
}

Received October 6, 2015; Accepted October 24, 2016

DOI: $10.3892 / \mathrm{mmr} .2016 .5980$

\begin{abstract}
Behcet's disease is a multi-system inflammatory disorder, and ocular Behcet's disease (OBD) is one of the most common causes of uveitis in China. A number of studies have indicated that Th17 cells, a subset of interleukin-17 (IL-17)-producing CD4+ T-helper cells, serve important roles in the pathogenesis of OBD. Berberine (BBR) is an isoquinoline derivative alkaloid isolated from Chinese herbs, and has been used traditionally for the treatment of gastrointestinal disorders. The aim of the present study was to investigate the effect of BBR on Th17 cell proliferation and cytokine secretion, and the expression and activation of the signal transducer and activator of transcription 3 (STAT3) transcription factor in OBD in vitro. Blood samples were obtained from healthy controls and patients with active ocular Behcet's disease. Peripheral blood mononuclear cells (PBMCs) or $\mathrm{CD}^{+} \mathrm{T}$ cells were cultured for three days with or without BBR and in the presence of anti-CD3 and anti-CD28 antibodies. IL-17 expression in cell sample supernatants was determined by enzyme-linked immunosorbent assay, and cell viability was measured using the Cell Counting kit- 8 assay. The number of $\mathrm{CD}^{+} \mathrm{IL}-17^{+}$cells and the expression level of phosphorylated (p)-STAT3 in $\mathrm{CD}^{+}{ }^{+} \mathrm{T}$ cells was determined using flow cytometry analysis. The expression of IL-17 was increased in patients with active OBD following the activation of PBMCs and $\mathrm{CD}^{+} \mathrm{T}$ cells with anti-CD3 and anti-CD28 antibodies
\end{abstract}

Correspondence to: Dr Zhuo Li, Department of Ophthalmology, The Second Xiangya Hospital, Central South University, 139 Middle Ren Min Road, Changsha, Hunan 410010, P.R. China

E-mail: zhuozhuoli@msn.com

Abbreviations: BBR, berberine; OBD, ocular Behcet's disease; PBMC, peripheral blood mononuclear cells; STAT3, signal transducer and activator of transcription 3; DMSO, Dimethyl sulfoxide; ELISA, Enzyme-linked immunosorbent assay; EAE, experimental autoimmune encephalomyelitis; EAU, experimental autoimmune uveitis; $\mathrm{HC}$, healthy controls; MFI, mean fluorescence intensity

Key words: berberine, ocular Behcet's disease, Th17, signal transducer and activator of transcription 3 when compared with healthy controls. However, no significant difference in cell viability following exposure to BBR was observed in PBMCs derived from healthy controls or patients with OBD. Following incubation with BBR, the expression of IL-17 was reduced and the number of $\mathrm{CD} 4^{+} \mathrm{IL}-17^{+}$cells was decreased in patients with active OBD and healthy controls. Furthermore, the expression of p-STAT3 was significantly decreased in the presence of BBR in healthy controls. In conclusion, the results of the present study demonstrate that BBR may suppress the Th17 response in patients with OBD by reducing STAT3 phosphorylation. BBR may be a potential therapeutic agent for the treatment of OBD.

\section{Introduction}

Berberine (BBR) is an isoquinoline derivative alkaloid isolated from Chinese herbs, such as those from the Berberis and Coptis genera (1). Aside from its traditional use as a treatment for gastrointestinal disorders, BBR has been demonstrated to exhibit inhibitory functions in a number of additional diseases, including cancer (2), microbial infection (3), inflammatory and autoimmune diseases (such as cardiovascular disease) (4), collagen-induced arthritis (5), experimental type I diabetes in mice (6) and experimental autoimmune encephalomyelitis (EAE) (7). Numerous reports have demonstrated that BBR may suppress the Th17 response in certain autoimmune diseases (6-8). However, it remains unclear whether BBR exerts this function by suppressing the Th17 response in Behcet's disease.

Behcet's disease is a multi-system inflammatory disorder characterized by recurrent uveitis, oral aphthae, genital ulcers or skin lesions (9). Ocular Behcet's disease (OBD) is one of the most common causes of uveitis in China (9). A number of studies have indicated that Th17 cells, a subset of interleukin-17 (IL-17)-producing $\mathrm{CD}^{+} \mathrm{T}$ helper cells, and their relevant cytokines, serve important roles in the pathogenesis of experimental autoimmune uveitis (EAU), as well as in clinical uveitis (including OBD) (10-12). IL-17, the characteristic cytokine produced by Th17 cells, has been observed to be upregulated in patients with active OBD, and neutralization of IL-17 using a specific antibody or through the inhibition of Th17 cells, led to the amelioration of EAU $(10,13)$. Corticosteroids and cyclosporin A, which are frequently used as effective immunosuppressive drugs to treat clinical uveitis, 
may significantly downregulate the Th17 cell response in patients with OBD (14).

The signal transducer and activator of transcription 3 (STAT3) serves a critical role in Th17 differentiation and IL-17 production (15). STAT3 activation is characterized by STAT3 phosphorylation, and previous studies have demonstrated that patients with active Behcet's disease exhibit elevated levels of STAT3 phosphorylation $(16,17)$.

The aim of the present study was to investigate the effect of BBR on the Th17 response in OBD. The results demonstrated that patients with active OBD exhibited an increased Th17 response. In vitro experiments revealed that BBR significantly downregulated the expression of IL-17 and the number of Th17 cells in patients with active OBD, and BBR could inhibit the phosphorylation of STAT3. This suggests that BBR may suppress intraocular inflammation in Behcet's disease, likely through downregulating the phosphorylation of STAT3, thereby inhibiting the Th17 response.

\section{Materials and methods}

$B B R$. BBR was purchased from Sigma-Aldrich; Merck Millipore (Darmstadt, Germany), and was dissolved in dimethyl sulfoxide (DMSO) before the stock solution $(50 \mathrm{mM})$ was stored at $-20^{\circ} \mathrm{C}$.

Patients and healthy controls. A total of 16 patients with active OBD, who were admitted to The Second Xiangya Hospital of Central South University (Changsha, China) from June 2014 to April 2015 (9 males and 7 females; average age, 34.8 years), and 18 healthy controls (HC; 10 males and 8 females; average age, 36.4 years) were included in the present study. Behcet's disease was diagnosed according to the diagnostic criteria of the International Study Group for Behcet's Disease (18). The patients presented with active uveitis, as evidenced by the presence of: i) Intraocular inflammation, which was characterized by the presence of floating cells in the anterior chamber or vitreous $(100 \%)$; and ii) retinal vasculitis, which was characterized by fundus fluorescein angiography (100\%). The extraocular systemic findings included recurrent oral aphthous ulcers (100\%), recurrent genital ulcers (43.8\%), skin lesions $(56.3 \%)$ and arthritis (18.8\%). The present study was approved by the Ethics Committee of the Second Xiangya Hospital of Central South University (Changsha, China). All procedures complied with the Declaration of Helsinki (19), and informed consent was obtained from all healthy individuals and patients with Behcet's disease that were included in the study.

Isolation and culture of cells. Anticoagulated whole blood samples were obtained from HCs and patients with active OBD. PBMCs were isolated using Ficoll-Hypaque density gradient centrifugation. Blood cells were diluted 1:1 with PBS. Ficoll-Hypaque solution (3-4 ml) was added into 15-ml centrifuge tubes, and the diluted blood cells $(6-8 \mathrm{ml})$ were slowly layered on top of the Ficoll-Hypaque solution. The tube was centrifuged at $4^{\circ} \mathrm{C}$ at $800 \times g$ for $25 \mathrm{~min}$ without the break. Following centrifugation, the PBMC layer appeared as a white, cloudy band between the blood cell and Ficoll-Hypaque layers, and was transferred to a new centrifuge tube and washed in PBS three times. Peripheral CD $4^{+} \mathrm{T}$ cells were isolated using human CD4 microbeads followed by magnetic-activated cell sorting according to the manufacturer's instructions (Miltenyi Biotec, Inc., San Diego, CA, USA). The purity of isolated cells identified by flow cytometry was $>94 \%$. PBMCs and CD $4^{+}$ $\mathrm{T}$ cells were resuspended in RPMI 1640 medium (Gibco; Thermo Fisher Scientific, Inc., Waltham, MA, USA) containing L-glutamine (2 $\mathrm{mM})$, penicillin/streptomycin $(100 \mathrm{U} / \mathrm{ml})$, and $10 \%$ fetal calf serum (Gibco; Thermo Fisher Scientific, Inc.), to a final concentration of $1 \times 10^{6}$ cells $/ \mathrm{ml}$. Cells were cultured for 3 days in the presence of BBR $(5 \mu \mathrm{M})$ or DMSO and anti-CD3 (catalog no. 130-093-387) and anti-CD28 (catalog no. 130-093-375) antibodies $(0.5$ and $0.1 \mu \mathrm{g} / \mathrm{ml}$, respectively; Miltenyi Biotec, Inc.) at $37^{\circ} \mathrm{C}, 100 \%$ humidity and $5 \% \mathrm{CO}_{2}$.

Enzyme-linked immunosorbent assay (ELISA). The levels of IL-17 in PBMCs and CD4 ${ }^{+} \mathrm{T}$ cell sample supernatants were measured using DuoSet ELISA Development Systems assay kits with a detection limit of $15.6 \mathrm{pg} / \mathrm{ml}$ according to the manufacturer's protocol (catalog no. DY317; R\&D Systems, Inc., Minneapolis, MN, USA).

Cell viability assay. The PBMC suspension was seeded onto a 96-well plate $\left(1 \times 10^{6}\right.$ cells $\left./ \mathrm{ml}\right)$, and treated with BBR $(5 \mu \mathrm{M})$ or DMSO, and cultured for 3 days in the presence of anti-CD3 $(0.5 \mu \mathrm{g} / \mathrm{ml})$ and anti-CD28 $(0.1 \mu \mathrm{g} / \mathrm{ml})$ antibodies. Cell viability was measured using the Cell Counting kit- 8 (Sigma-Aldrich; Merck Millipore). Absorbance was read at $450 \mathrm{~nm}$ using an ELISA plate reader.

Flow cytometry. $\mathrm{CD}^{+} \mathrm{T}$ cells were cultured in the presence of BBR or DMSO, and anti-CD3 $(0.5 \mu \mathrm{g} / \mathrm{ml})$ and anti-CD28 antibodies $(0.1 \mu \mathrm{g} / \mathrm{ml})$. In order to determine the number of $\mathrm{IL}-17^{+}$cells, CD4 ${ }^{+} \mathrm{T}$ cell samples were stimulated with phorbol 12-myristate 13 -acetate $(100 \mathrm{ng} / \mathrm{ml})$ and ionomycin (1 $\mu \mathrm{g} / \mathrm{ml}$; Sigma-Aldrich; Merck Millipore) for $5 \mathrm{~h}$. During the final $4 \mathrm{~h}$, brefeldin A $(10 \mu \mathrm{g} / \mathrm{ml}$; Sigma-Aldrich; Merck Millipore) was added. Fixation/Permeabilization Concentrate (1 part; catalog no. 00-5123-43; eBioscience; Affymetrix, Inc., Santa Clara, CA, USA) was mixed with Fixation/Permeabilization Diluent (3 parts; catalog no. 00-5223-56; eBioscience; Affymetrix, Inc.), and $1 \mathrm{ml}$ of this Fixation/Permeabilization solution was added to stimulated cells for $30 \mathrm{~min}$ at $4^{\circ} \mathrm{C}$. Subsequently, $2 \mathrm{ml} 1 \mathrm{X}$ Permeabilization Buffer (catalog no. 00-8333; eBioscience; Affymetrix, Inc.) was added to wash the cells. Cells were resuspended in $100 \mu \mathrm{l} 1 \mathrm{X}$ Permeabilization Buffer, and stained with a fluorescein isothiocyanate (FITC)-conjugated mouse anti-human IL-17 antibody (catalog no. 11-7179-41; eBioscience; Affymetrix, Inc.) or a FITC-conjugated mouse IgG1 $\kappa$ isotype control (catalog no. 11-4714-41; eBioscience; Affymetrix, Inc.). The IL- $17^{+}$cells in the $\mathrm{CD} 4^{+} \mathrm{T}$ cell population were defined as Th17 cells.

Detection of phosphorylated (p)-STAT3 expression in $\mathrm{CD}^{+} \mathrm{T}$ cells was achieved by culturing cells in the presence of BBR $(5 \mu \mathrm{M})$ or DMSO, together with anti-CD3 $(0.5 \mu \mathrm{g} / \mathrm{ml})$ and anti-CD28 antibodies $(0.1 \mu \mathrm{g} / \mathrm{ml})$ for $30 \mathrm{~min}$. Cells were subsequently fixed and permeabilized as described above, and stained with a phycoerythrin (PE)-conjugated mouse anti-human pSTAT3 antibody (catalog no. 558557; BD Biosciences, San Jose, CA, USA) or a PE-conjugated mouse 

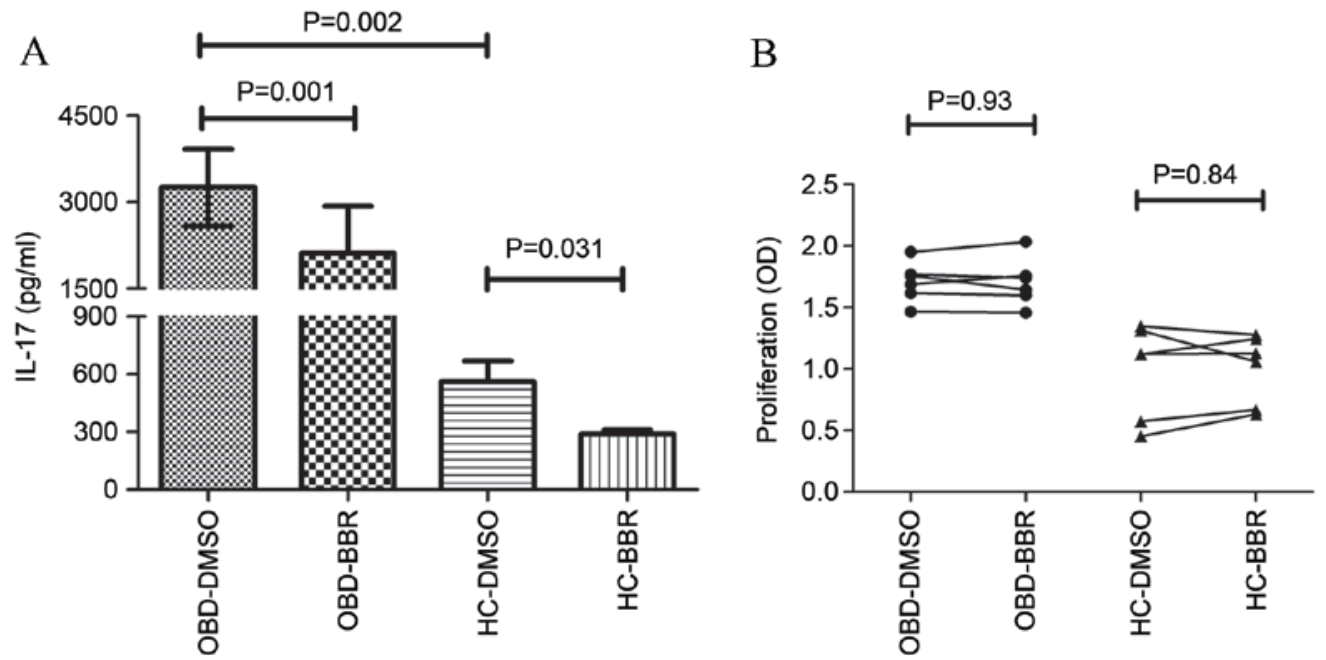

Figure 1. Effect of BBR on PBMC IL-17 production and cell viability. (A) The expression of IL-17 in PBMCs derived from HC ( $\mathrm{n}=6$ ) and patients with active OBD $(n=6)$ was significantly decreased in the presence of BBR $(5 \mu \mathrm{M})$ compared with DMSO, as determined by flow cytometry analysis. (B) No significant difference in PBMC cell viability was observed between $\mathrm{HC}(\mathrm{n}=6)$ and $\mathrm{OBD}(\mathrm{n}=6)$ groups. Data are presented as the mean \pm standard deviation. BBR, berberine; IL-17, interleukin 17; PBMC, peripheral blood mononuclear cells; HC, healthy control; OBD, ocular Behcet's disease; DMSO, dimethyl sulfoxide.

IgG1 isotype control (catalog no. 12-4714; eBioscience; Affymetrix, Inc.).

Flow cytometry analysis was performed using a FACSAria cytometer (BD Biosciences) and analyzed using FlowJo software (Tree Star, Inc., San Carlos, CA, USA).

Statistical analysis. Data are expressed as the mean \pm standard deviation. Statistical analysis was performed using SPSS (version 13.0; SPSS, Inc., Chicago, IL, USA). Differences between sample groups were analyzed using Student's t-test, Mann-Whitney U-test and the Wilcoxon signed-rank test. $\mathrm{P}<0.05$ was considered to indicate a statistically significant difference.

\section{Results}

BBR inhibited the production of $I L-17$ but had no effect on the viability of PBMCs. PBMCs obtained from $\mathrm{HC}$ and patients with active OBD were cultured in the presence of BBR $(5 \mu \mathrm{M})$ or DMSO, together with anti-CD3 and anti-CD28 antibodies for 3 days, in order to investigate the effect of BBR on IL-17 production. Patients with active OBD demonstrated a significant increase in IL-17 expression compared with HC $(3,251.98 \pm 666.02$ vs. $561.61 \pm 108.37 \mathrm{pg} / \mathrm{ml} ; \mathrm{P}=0.002$; Fig. $1 \mathrm{~A})$. As shown in Fig. 1A, BBR significantly inhibited the expression of IL-17 in PBMCs from patients with active OBD (from $3,251.98 \pm 666.02$ to $2,113.63 \pm 815.07 \mathrm{pg} / \mathrm{ml} ; \mathrm{P}=0.0001)$ and $\mathrm{HC}$ (from $561.61 \pm 108.37$ to $287.66 \pm 23.67 \mathrm{pg} / \mathrm{ml} ; \mathrm{P}=0.031$ ). In order to investigate whether BBR inhibited IL-17 production by reducing PBMC cell viability, the effect of BBR exposure on PBMC cell viability was then examined. As shown in Fig. 1B, BBR did not have a significant effect on the viability of PBMC cells in $\mathrm{HC}$ or OBD groups at a concentration of $5 \mu \mathrm{M}$.

BBR inhibited the production of IL-17 in $C D 4^{+} T$ cells from $H C s$ and patients with active $O B D$. The expression of IL-17 in $\mathrm{CD}^{+} \mathrm{T}$ cells was significantly higher upon stimulation with anti-CD3 and anti-CD28 antibodies in patients with active OBD

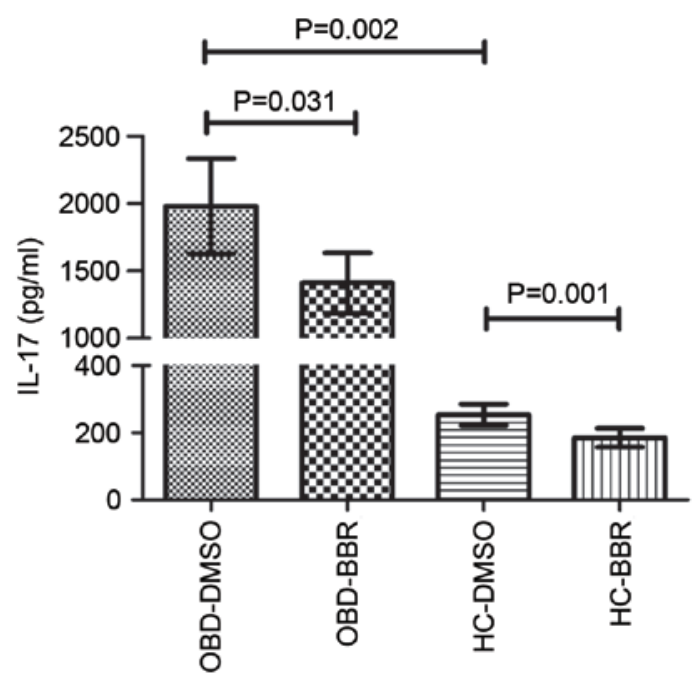

Figure 2. Effect of BBR on IL-17 expression in $\mathrm{CD}^{+} \mathrm{T}$ cells. In the presence of BBR, the expression of IL-17 in $\mathrm{CD}^{+} \mathrm{T}$ cells from $\mathrm{HCs}(\mathrm{n}=6)$ and patients with active OBD $(n=6)$ was significantly reduced compared with DMSO-treated cells. Data are presented as the mean \pm standard deviation. BBR, berberine; IL-17, interleukin 17; HC, healthy control; OBD, ocular Behcet's disease; DMSO, dimethyl sulfoxide.

compared with HCs $(1,981.73 \pm 869.59$ vs. $253.31 \pm 77.15 \mathrm{pg} / \mathrm{ml}$; $\mathrm{P}=0.002$; Fig. 2). Exposure to BBR significantly decreased the production of IL-17 by $\mathrm{CD} 4^{+} \mathrm{T}$ cells in active OBD patients (from $1,981.73 \pm 869.59$ to $1,410.39 \pm 545.41 \mathrm{pg} / \mathrm{ml} ; \mathrm{P}=0.031$ ) and HCs (from $253.31 \pm 77.15$ to $185.11 \pm 67.80 \mathrm{pg} / \mathrm{ml} ; \mathrm{P}=0.001$; Fig. 2).

BBR inhibited the number of Th17 cells in patients with active $O B D$ and healthy controls. As shown in Fig. 3, the number of Th17 cells (IL-17-producing CD4+ $\mathrm{T}$ cells) was significantly higher in patients with active OBD compared with HCs $(5.75 \pm 0.88$ vs. $39 \pm 0.43 \%$; $\mathrm{P}<0.001)$. In addition, exposure to BBR significantly inhibited the number of Th17 cells in patients with active OBD (from $5.75 \pm 0.88$ to $4.42 \pm 0.82 \%$; 
A

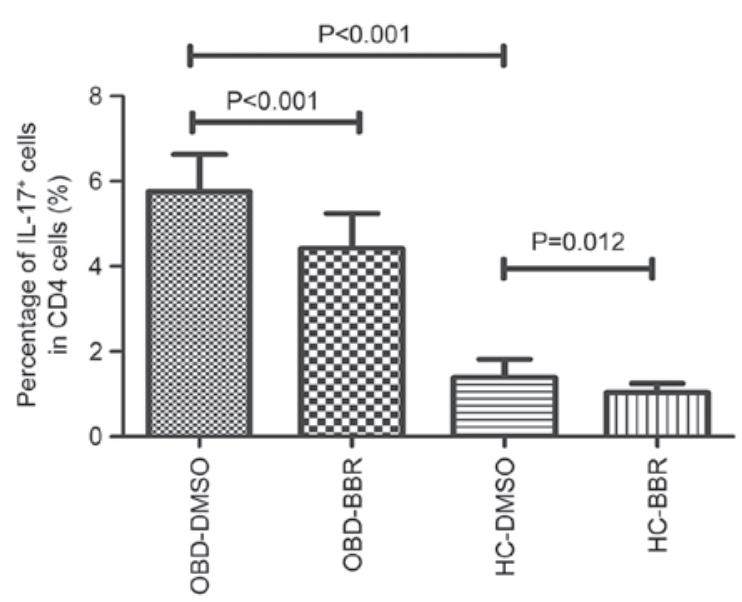

B
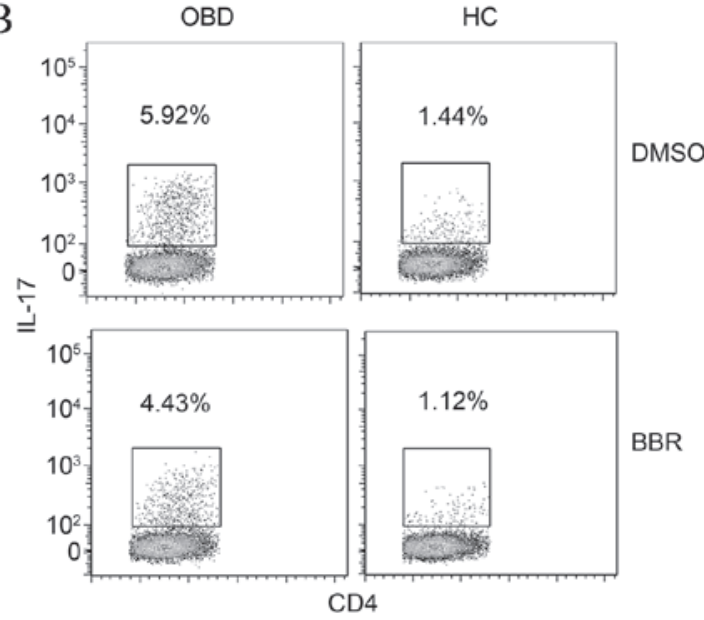

Figure 3. Effect of BBR on the number of Th17 (IL-17+) cells. (A) The results represent the percentages of IL-17 $7^{+}$cells among CD4 ${ }^{+}$T cells obtained from HCs and patients with active OBD, following treatment with BBR or DMSO. (B) Representative plots of IL-17 $7^{+}$cells in a HC and a patient with OBD. The percentage of IL- $17^{+}$cells among CD4+ cells is indicated in each plot. Data are presented as the mean \pm standard deviation. BBR, berberine; IL-17, interleukin 17 ; HC, healthy control; OBD, ocular Behcet's disease; DMSO, dimethyl sulfoxide.

$\mathrm{P}<0.001$ ) and $\mathrm{HCs}$ (from $1.39 \pm 0.43$ to $1.03 \pm 0.22 \% ; \mathrm{P}=0.012$ Fig. 3).

BBR inhibited the activation of STAT3. Due to the observation that STAT3 activation is an important factor involved in the differentiation of Th17 cells and the production of IL-17 (15), the effect of BBR on modulating STAT3 phosphorylation was investigated. As shown in Fig. $4 \mathrm{~A}$ and $\mathrm{B}$, exposure of $\mathrm{CD}^{+}$ $\mathrm{T}$ cells, obtained from HCs, to BBR significantly decreased the number of p-STAT3-positive cells from 96.6 \pm 1.24 to $93.9 \pm 0.54 \%(\mathrm{P}=0.0 .003)$. The expression of $\mathrm{p}-\mathrm{STAT} 3$ was then determined using the mean fluorescence intensity (MFI) parameter. The results demonstrated that the MFI value of p-STAT3 expression in BBR-treated $\mathrm{CD}^{+} \mathrm{T}$ cells was significantly lower compared with that of the DMSO-treated cells (506.67 \pm 32.75 vs. $396.33 \pm 58.49$; $\mathrm{P}<0.001$; Fig. 4C).

\section{Discussion}

In the present study the potential role of BBR as a potential therapeutic agent for OBD was investigated. The results demonstrated that BBR suppressed IL-17 expression and the number of Th17 cells in PBMCs obtained from patients with active $\mathrm{OBD}$ and $\mathrm{HCs}$. In addition, BBR inhibited the activation of STAT3. These results suggest that BBR may suppress the Th17 response in patients with OBD by downregulating the phosphorylation of STAT3, and therefore provides a rationale for investigating the potential use of BBR for the treatment of patients with $\mathrm{OBD}$ in a clinical setting.

BBR is an isoquinoline alkaloid isolated from certain Chinese herbs, such as Berberis, Hydrastis canadensis and Coptidis rhizoma (1). The role of BBR in the treatment of a number of inflammatory and autoimmune disorders has been reported previously $(4,6,7,20,21)$. In addition, the role of BBR in uveitis has been investigated; in endotoxin-induced uveitis, Berberis aristata, whose primary components include BBR, inhibited ocular inflammation (22). Furthermore, BBR demonstrated an immunoregulatory role in the Vogt-Koyanagi-Harada ocular autoimmune disease (8). These studies support a role for BBR as an anti-inflammatory agent.

As Th17 cell-associated inflammatory responses are critically involved in the pathogenesis of OBD (23), the present study was designed to investigate the effect of BBR on the function of Th17 cells in OBD. The effect of BBR on the secretion of IL-17 by PBMCs was first investigated. Consistent with Chi et al (24), a significant increase in the expression of IL-17 was observed in patients with active OBD compared with HCs. Following exposure to BBR, IL-17 expression in PBMCs was significantly suppressed. Cell viability is an important parameter that can influence cytokine production (25). Therefore, we investigated whether BBR-mediated suppression of IL-17 expression was due to the suppression of T-cell viability. The results demonstrated that BBR did not influence $\mathrm{T}$ cell viability when exposed to a concentration of $5 \mu \mathrm{M}$. This result is largely consistent with a previous report, in which $50 \mu \mathrm{M}$ BBR did not increase the death rate of lymphocytes (26).

The results of the present study indicate a suppressive effect of BBR on the secretion of IL-17 by PBMCs. As Th17 cells primarily produce IL-17, CD $4^{+} \mathrm{T}$ cells were purified from PBMCs in order to verify the suppressive effect of BBR on IL-17 secretion by Th17 cells. The results revealed that BBR may inhibit the secretion of IL-17 by Th17 cells.

Previous studies have demonstrated that STAT3 activation is critical for Th17 differentiation and IL-17 production (15). STAT3 activation is characterized by its phosphorylation, which leads to the regulation of downstream target genes that mediate intrinsic and extrinsic cellular functions (15). In the present study STAT3 phosphorylation was significantly inhibited in $\mathrm{CD}^{+} \mathrm{T}$ cells following BBR exposure, which may have been responsible for the suppressive effect of BBR on the function of Th17 cells. This result is consistent with those presented by Qin et al (7), which used a mouse model of EAE, and demonstrated that Th17 cell differentiation was markedly inhibited by BBR treatment in vivo and in vitro, and that this was associated with decreased phosphorylation of STAT3 in Th17 cells. 
A
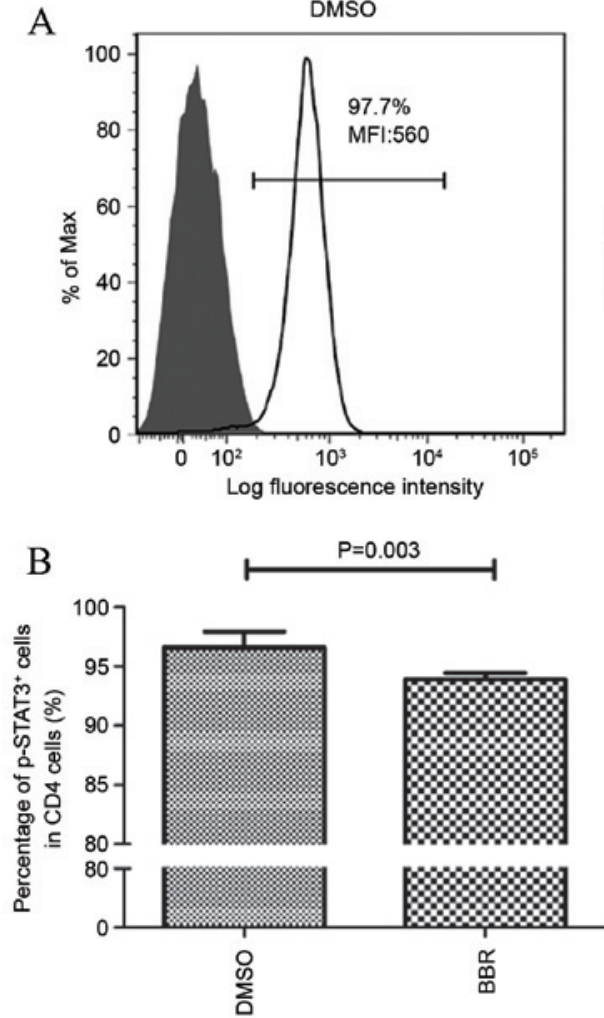

BBR
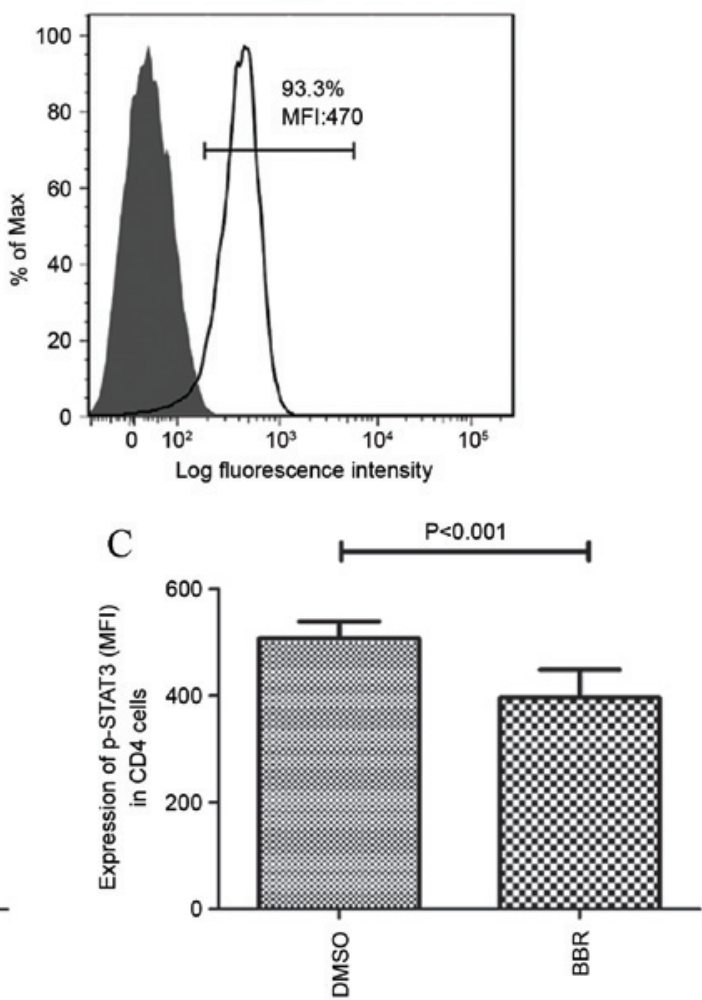

Figure 4. Effects of BBR on STAT3 activation. (A) Representative histograms illustrate the expression of p-STAT3 in anti-CD3/28-stimulated CD4+ ${ }^{+}$cells in a HC treated with DMSO or BBR. (B) The number of p-STAT3 ${ }^{+}$cells expressed as a percentage of CD4 ${ }^{+} \mathrm{T}$ cells from $\mathrm{HC}$ following exposure to BBR or DMSO. (C) The expression of p-STAT3 expressed as the mean fluorescence intensity (MFI) in CD4 ${ }^{+} \mathrm{T}$ cells from HCs following exposure to BBR or DMSO. Data are presented as the mean \pm standard deviation. BBR, berberine; p-STAT3, phosphorylated signal transducer and activator of transcription 3; DMSO, dimethyl sulfoxide; HC, healthy control.

Despite these observations, a number of important questions for the use of BBR as a potential treatment for OBD remain. Namely, the selection of a safe but effective dose of BBR for the treatment of OBD. Our results demonstrate that BBR is not a cytotoxic or a general immunosuppressive agent that affects $\mathrm{T}$ cell survival in vitro. In addition, BBR is currently used for the treatment of numerous gastrointestinal diseases in China, and to the best of our knowledge, no unexpected safety concerns have yet been identified. A recent randomized, placebo-controlled, double-blind trial concerning the effects of BBR gelatin on recurrent aphthous stomatitis, has demonstrated that the use of BBR gelatin is safe and effective (27). Although there is no evidence to suggest that increasing the dose of BBR may cause severe adverse effects, there have been no reports on the safety of BBR treatment in large doses and in the long-term, thus, the appropriate dose of BBR for the treatment of OBD in vivo remains a critical factor that requires further investigation. In addition, the concentration of BBR in the plasma is an important aspect for improving the symptoms of autoimmune disease (28). Therefore further studies are required to define the optimal plasma BBR concentrations for the treatment of $\mathrm{OBD}$ in vivo.

Another important consideration for the use of BBR as a treatment for OBD, is based on the observation that BBR did not completely suppress the Th17 cell response in the present study. According to these results, the decreased levels of IL-17 expression and Th17 cell number following exposure of PBMCs obtained from OBD patients to BBR, were not as low as observed in the HC group. This suggests that BBR treatment alone may be insufficient to control the disease. Therefore, further studies are required to investigate whether BBR in combination with other currently used immunosuppressive agents may modulate an aberrant immune response, and may be a more suitable therapeutic approach for the treatment of patients with OBD and additional autoimmune diseases mediated by an abnormal Th17 immune response.

In conclusion, the results of the present study indicate that BBR exhibits an inhibitory effect on the Th17 cell response. BBR-mediated suppression of STAT3 activation abrogates downstream signaling events, and results in the decreased expression of IL-17 and the number of Th17 cells, which is one of the potential mechanisms underlying the immunosuppressive effects of BBR.

\section{Acknowledgements}

The authors would like to thank all of the patients and healthy donors enrolled in the study. The present study was supported by the Development Program of Hunan Provincial Science \& Technology Department (grant no. 2014FJ3006).

\section{References}

1. Ye M, Fu S, Pi R and He F: Neuropharmacological and pharmacokinetic properties of berberine: A review of recent research. J Pharm Pharmacol 61: 831-837, 2009. 
2. Song YC, Lee Y, Kim HM, Hyun MY, Lim YY, Song KY and Kim BJ: Berberine regulates melanin synthesis by activating PI3K/AKT, ERK and GSK3 $\beta$ in B16F10 melanoma cells. Int J Mol Med 35: 1011-1016, 2015.

3. Saha P, Bhattacharjee S, Sarkar A, Manna A, Majumder S and Chatterjee M: Berberine chloride mediates its anti-leishmanial activity via differential regulation of the mitogen activated protein kinase pathway in macrophages. PLoS One 6: e18467, 2011.

4. Chi L, Peng L, Pan N, Hu X and Zhang Y: The anti-atherogenic effects of berberine on foam cell formation are mediated through the upregulation of sirtuin 1. Int J Mol Med 34: 1087-1093, 2014.

5. Hu Z, Jiao Q, Ding J, Liu F, Liu R, Shan L, Zeng H, Zhang J and Zhang W: Berberine induces dendritic cell apoptosis and has therapeutic potential for rheumatoid arthritis. Arthritis Rheum 63: 949-959, 2011.

6. Cui G, Qin X, Zhang Y, Gong Z, Ge B and Zang YQ: Berberine differentially modulates the activities of ERK, p38 MAPK, and JNK to suppress Th17 and Th1 T cell differentiation in type 1 diabetic mice. J Biol Chem 284: 28420-28429, 2009.

7. Qin X, Guo BT, Wan B, Fang L, Lu L, Wu L, Zang YQ and Zhang JZ: Regulation of Th1 and Th17 cell differentiation and amelioration of experimental autoimmune encephalomyelitis by natural product compound berberine. J Immunol 185: 1855-1863, 2010.

8. Yang Y, Qi J, Wang Q, Du L, Zhou Y, Yu H, Kijlstra A and Yang P: Berberine suppresses Th17 and dendritic cell responses. Invest Ophthalmol Vis Sci 54: 2516-2522, 2013.

9. Yang P, Fang W, Meng Q, Ren Y, Xing L and Kijlstra A: Clinical features of chinese patients with Behçet's disease. Ophthalmology 115: 312-318, 2008.

10. Amadi-Obi A, Yu CR, Liu X, Mahdi RM, Clarke GL, Nussenblatt RB, Gery I, Lee YS and Egwuagu CE: TH17 cells contribute to uveitis and scleritis and are expanded by IL-2 and inhibited by IL-27/STAT1. Nat Med 13: 711-718, 2007.

11. Chi W, Yang P, Li B, Wu C, Jin H, Zhu X, Chen L, Zhou H, Huang $X$ and Kijlstra A: IL-23 promotes CD4 ${ }^{+}$T cells to produce IL-17 in Vogt-Koyanagi-Harada disease. J Allergy Clin Immunol 119: 1218-1224, 2007.

12. Peng Y, Han G, Shao H, Wang Y, Kaplan HJ and Sun D Characterization of $\mathrm{IL}_{-1} 7^{+}$interphotoreceptor retinoid-binding protein-specific $\mathrm{T}$ cells in experimental autoimmune uveitis. Invest Ophthalmol Vis Sci 48: 4153-4161, 2007.

13. Luger D, Silver PB, Tang J, Cua D, Chen Z, Iwakura Y, Bowman EP, Sgambellone NM, Chan CC and Caspi RR: Either a Th17 or a Th1 effector response can drive autoimmunity: Conditions of disease induction affect dominant effector category. J Exp Med 205: 799-810, 2008.

14. Liu X, Yang P, Lin X, Ren X, Zhou H, Huang X, Chi W, Kijlstra A and Chen L: Inhibitory effect of Cyclosporin A and corticosteroids on the production of IFN-gamma and IL-17 by $\mathrm{T}$ cells in Vogt-Koyanagi-Harada syndrome. Clin Immunol 131: $333-342,2009$
15. Mathur AN, Chang HC, Zisoulis DG, Stritesky GL, Yu Q, O'Malley JT, Kapur R, Levy DE, Kansas GS and Kaplan MH: Stat3 and Stat4 direct development of IL-17-secreting Th cells. J Immunol 178: 4901-4907, 2007.

16. Tulunay A, Dozmorov MG, Ture-Ozdemir F, Yilmaz V, Eksioglu-Demiralp E, Alibaz-Oner F, Ozen G, Wren JD, Saruhan-Direskeneli G, Sawalha AH and Direskeneli $\mathrm{H}$ : Activation of the JAK/STAT pathway in Behcet's disease. Genes Immun 16: 170-175, 2014.

17. Qi J, Yang Y, Hou S, Qiao Y, Wang Q, Yu H, Zhang Q, Cai T, Kijlstra A and Yang P: Increased Notch pathway activation in Behçet's disease. Rheumatology (Oxford) 53: 810-820, 2014.

18. Criteria for diagnosis of Behcet's disease. International Study Group for Behçet's Disease. Lancet 335: 1078-1080, 1990.

19. Rickham PP: Human experimentation. Code of ethics of the world medical association. Declaration of Helsinki. Br Med J 2: 177,1964

20. Ma X, Jiang Y, Wu A, Chen X, Pi R, Liu M and Liu Y: Berberine attenuates experimental autoimmune encephalomyelitis in C57 BL/6 mice. PLoS One 5: e13489, 2010.

21. Marinova EK, Nikolova DB, Popova DN, Gallacher GB and Ivanovska ND: Suppression of experimental autoimmune tubulointerstitial nephritis in $\mathrm{BALB} / \mathrm{c}$ mice by berberine. Immunopharmacology 48: 9-16, 2000.

22. Gupta SK, Agarwal R, Srivastava S, Agarwal P, Agrawal SS Saxena R and Galpalli N: The anti-inflammatory effects of Curcuma longa and Berberis aristata in endotoxin-induced uveitis in rabbits. Invest Ophthalmol Vis Sci 49: 4036-4040, 2008.

23. Na SY, Park MJ, Park S and Lee ES: Up-regulation of Th17 and related cytokines in Behçet's disease corresponding to disease activity. Clin Exp Rheumatol 31: (3 Suppl 77) 32-40, 2013.

24. Chi W, Yang P, Zhu X, Wang Y, Chen L, Huang X and Liu X: Production of interleukin-17 in Behcet's disease is inhibited by cyclosporin A. Mol Vis 16: 880-886, 2010.

25. Hedegaard CJ, Krakauer M, Bendtzen K, Sorensen PS, Sellebjerg $\mathrm{F}$ and Nielsen $\mathrm{CH}$ : The effect of beta-interferon therapy on myelin basic protein-elicited $\mathrm{CD}^{+} \mathrm{T}$ cell proliferation and cytokine production in multiple sclerosis. Clin Immunol 129: 80-89, 2008.

26. Xu L, Liu Y and He X: Inhibitory effects of berberine on the activation and cell cycle progression of human peripheral lymphocytes. Cell Mol Immunol 2: 295-300, 2005.

27. Jiang XW, Zhang Y, Zhu YL, Zhang H, Lu K, Li FF and Peng HY: Effects of berberine gelatin on recurrent aphthous stomatitis: A randomized, placebo-controlled, double-blind trial in a Chinese cohort. Oral Surg Oral Med Oral Pathol Oral Radiol 115: 212-217, 2013.

28. Pang B, Zhao LH, Zhou Q, Zhao TY, Wang H, Gu CJ and Tong XL: Application of berberine on treating type 2 diabetes mellitus. Int J Endocrinol 2015: 905749, 2015. 